\title{
ONE-PARAMETER PROJECTIVE GROUPS AND THE CLASSIFICATION OF COLLINEATIONS*
}

BY

\author{
EDWARD B. VAN VLECK
}

The origin of the following paper is to be found in the application of the iterative principle to the classification of collineations. To this $I$ have been unexpectedly led by my preceding paper on the Extension of a Theorem of Poincaré. Other topics closely connected with my subject which will be treated here are as follows:

1. The determination of a normal form for one-parameter collineation groups in $n$ homogeneous variables, also for homogeneous linear groups in $n$ variables.

The form consists namely of components of the structure (3) below, where $m$ is the parameter of the group. The total number of equations of all the components must, of course, be equal to $n$.

2. The determination of a real normal form for real groups of the character above described.

It consists of components of form (3), in which $\rho$ is to be taken positive, and of components of the form (14).

3. The enumeration and classification of these groups for any value of $n$; in particular, for $n=3,4$.

4. The varieties and form of the real path curves (the so-called $W$-curves) for real one-parameter collineation groups in space of three dimensions. The quadratic complex of tangents is also briefly considered.

The prevalent mode of classifying collineations has been developed by SEGRE and others and is based upon the normal form into which the finite equations of collineation may be transformed in accordance with the theory of elementary divisors. Geometrically, this form is dependent upon the invariantive configuration of points, lines, planes, etc. LIE, on the other hand, uses the infinitesimal transformation for classification. $\dagger$ The results correspond to those obtained by the preceding method. An obvious inconvenience or difficulty arises when a collineation is given, as is most commonly

\footnotetext{
* Presented under a slightly different title at the Chicago meeting of the Society, April 29, 1911.

†Cf., for example, LIE-Scherfrers, Vorlesungen über continuierliche Gruppen, pp. 65, 290291.
} 
the case, in finite and not in infinitesimal form. So far as I am aware, there has not existed prior to the present paper any criterion or formula giving the infinitesimal transformation, or transformations by which a given finite collineation may be generated - for example, a collineation with given numerical coefficients. For the case of plane collineations a passage from the given finite form to the infinitesimal transformation was effected as long ago as 1871 by KLEIN and LIE,* but their method has not been developed for space of higher dimensions.

LIE's work also opens up the possibility of another mode of classification based upon the character of the system of path curves to which an infinitesimal transformation gives rise. This method has been largely ignored; indeed, I do not feel sure that anyone has had expressly in mind the use of path curves for the purpose of classifying collineations. Though less direct, this method would have the advantage of being more discriminating than either of the two preceding methods. For example, while the method of SEGRE gives five types of plane collineations, this method at once subdivides one of his five types into three, $\dagger$ thus yielding seven distinct types in all. A complete classification of the path-curve systems in space $S_{3}$, and correspondingly of space collineations, has not been given hitherto, though most interesting properties of some of these curve systems have been given by KLEIN and LIE.f

The method of classification which I shall give here has the same discriminating power as the standpoint last mentioned, yet retains the advantages of the method of SEGRE. My starting point is, in fact, his familiar classification. The various types of collineations are iterated indefinitely, when differences arise between collineations of the same type, thus giving a further subdivision into classes. The iteration supplemented by functional interpolation also furnishes a one-parameter collineation group and leads to a very simple solution of the following problem: Given a finite collineation in $n$ homogeneous variables, to set up in finite form the continuous one-parameter group (or groups) in which it is contained. Thereby the classification of collineations becomes parallel to the classification of these groups or of the accompanying system of path curves, except for the very restricted set of cases in which a collineation can be included in several groups.

* Mathematische Annalen, vol. 4, p. 50. See also BOcher, Bulletin of the New York Mathematical Society, vol. 1 (1892), p. 225.

† Namely, the type with three invariant points. In LiE-ScHeFrers (loc. cit., pp. 77-79) a distinction of cases is made according as the three invariant points are real or one real and the other two conjugate imaginaries. In the latter case the path curves are in general logarithmic spirals. No note is there made of the fact that a seventh case arises when the system of spirals degenerates into a system of concentric circles, although the existence of this case is obvious from the equations given. This failure to specifically point out the existence of a seventh case is indicative of the general neglect of the application of Lie's path curves to the classification of collineations.

$\ddagger$ Comptes Rendus, vol. 70 (1870), pp. 1222, 1275. 
Since reaching my result I have found that precisely the same iterative method was previously applied in a very well known memoir by KLEIN and LIE $^{*}$ to pass from the five types of plane collineations to the corresponding continuous one-parameter groups. Indeed, it seems to have been precisely this which led to the introduction of the infinitesimal transformation. $\dagger$ It seems therefore rather strange that the iterative principle has not been similarly applied to the general case of $n$ variables. Possibly the discovery and development of the infinitesimal transformation with its wealth of applications diverted work from this direction. Possibly also the generalization may have been overlooked because the plane collineations iterated by KLEIN and LIE were expressed in unhomogeneous variables and not in the standard homogeneous form in which they are given today by the theory of elementary divisors.

It is to be observed that the program here brought forward for one-parameter collineation groups is the reverse of that of LIE. The method of LIE, on the one hand, starts with the infinitesimal transformation and ascends by integration, etc., to continuous gr.oups of finite collineations. .Here, on the other hand, a single finite collineation is taken as the starting point, from which the continuous group and infinitesimal transformation are to be derived. The normal form obtained for the group conducts at once to the complete enumeration of collineation groups in finite form for any number of homogeneous variables, a problem which seems to have been solved hitherto only for the cases of two and of three variables.

$\mathrm{My}$ formulas for the continuous groups give also the parametric representation of the corresponding systems of $W$-curves. A brief study is made of the different classes of real $W$-curves in $S_{3}$, some of which are new and very interesting.

For the particular case of the collineation of a straight line into itself, given by the formula

$$
x^{\prime}=\frac{a x+b}{c x+d}
$$

we have in the literature the familiar division into parabolic, elliptic, hyperbolic and loxodromic types. These distinctions arise from the interpretation of the imaginary path curves upon the line as real curves in the complex plane of $x$. The classification of collineations which $\mathrm{I}$ have introduced here may be regarded as essentially a corresponding classification for any number of variables, though no effort has been made to interpret unreal path curves in $n$-dimensions as real path curves in $2 n$-dimensional space. Such an inter-

* Mathematische Annalen, vol. 4 (1871), p. 50.

†Cf. Mathematische Annalen, vol. 53 (1900), pp. 6-8. In his review of LIE's work NoEther expresses his regret that the program of KLEIN and Lie for three-dimensional collineations sketched in the Comptes Rendus, was not later completed, as was projected. 
pretation would lead to a subdivision of some of my classes in correspondence with the cleavage between the hyperbolic and loxodromic types in the complex plane. The two latter conduct by iteration any point of the complex $x$-plane to one of the two invariant points as a limit, but are commonly differentiated from one another because of the differences in the system of path curves to which they give rise.

It may be useful in this connection to refer the reader to some recent work by J. Wesley Young.* By the use of " chains" Young extends the notion of path curves in the complex plane of $x$ in a very different manner to the case of two complex variables.

\section{Part I. The one-Parameter collineation group.}

\$1. The standard form for a collineation.-Consider any linear substitution with non-vanishing determinant given by the formula:

$$
\begin{aligned}
x_{1}^{\prime} & =a_{11} x_{1}+\cdots+a_{1 n} x_{n}, \\
\cdot & \cdot \cdot \cdot \cdot \cdot \cdot \cdot \cdot \\
x_{n}^{\prime} & =a_{n 1} x_{1}+\cdots+a_{n n} x_{n} .
\end{aligned}
$$

This will be interpreted at pleasure either as the general representation of a collineation expressed in $n$ homogeneous variables or as a homogeneous collineation in space of $n$ dimensions.

Suppose now that $C^{\prime}$ is transformed by application of a second linear homogeneous transformation $T$ in $n$ variables with non-vanishing determinant. The theory of elementary divisors shows that by properly choosing $T$ the transformed substitution $C=T C^{\prime} T^{-1}$ can be brought into simplified standard form. In this form the variables of $C$ are separated into independent sets, only the variables within a set being connected with one another. If the variables of a set are denoted by $x_{1}, x_{2}, \cdots, x_{r}$, the equations connecting them have the character:

$$
\begin{aligned}
& x_{1}^{\prime}=\rho x_{1}, \\
& x_{2}^{\prime}=x_{1}+\rho x_{2}, \\
& x_{3}^{\prime}=x_{2}+\rho x_{3}, \\
& \cdot \cdot \cdot \cdot \cdot \cdot \\
& x_{r}^{\prime}=x_{r-1}+\rho x_{r}
\end{aligned}
$$

Thus the substitution (collineation) is resolved into component substitutions (collineations) which may be considered in entire independence of one another. I shall call $r$ the order of the component. The sum of the orders is equal to $n$.

* These Trans a c ti on s, vol. 11 (1910), p. 280. On the basis of his chains Youva distinguishes six classes of plane collineations, one of which could have been readily split into two. 
The values of $\rho$ in the component substitutions are the roots of the characteristic equation

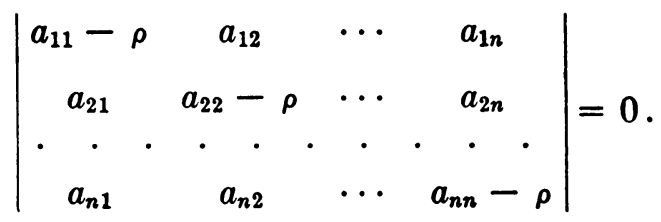

To each elementary divisor $\left(x-\rho_{i}\right)^{r}$ of this determinant there will correspond a component of order $r$. If for a multiple root $\rho_{i}$ there are several elementary divisors, there will be an equal number of component substitutions having the same value of $\rho_{i}$. Inasmuch as the elementary divisors are not altered when $C^{\prime}$ is transformed through $T$, the standard form obtained for $C$ is unique; but there is sometimes the possibility of reaching it by application of essentially distinct transformations $T$, or, in other words, by different changes of variable $T$.

$\S 2$. Iteration of the collineation.-Suppose now the original collineation $C^{\prime}$ to be repeated $m$ times. Then

$$
C^{m}=T^{-1} C^{m} T \text {. }
$$

Thus the problem of iterating $C^{\prime}$ is equivalent to that of iterating $C$, the result in either case being obtained from that in the other by transformation through $T$ or its inverse.

Consider then the effect of repeating $m$ times a component collineation (1). The result is given by the equations:

$$
\begin{aligned}
x_{1}^{\prime} & =\rho^{m} x_{1}, \\
x_{2}^{\prime} & =m \rho^{m-1} x_{1}+\rho^{m} x_{2 s} \\
x_{3}^{\prime} & =\frac{m(m-1)}{2 !} \rho^{m-2} x_{1}+m \rho^{m-1} x_{2}+\rho^{m} x_{3}, \\
& \cdot \cdot \cdot \cdot \cdot \cdot \cdot \cdot \cdot \cdot \cdot \cdot \cdot \\
x_{r}^{\prime} & =\frac{m(m-1) \cdots(m-r+2)}{(r-1) !} \rho^{m-r+1} x_{1} \\
& \quad+\frac{m(m-1) \cdots(m-r+3)}{(r-2) !} \rho^{m-r+2} x_{2}+\cdots+\rho^{m} x_{r} .
\end{aligned}
$$

This can be established immediately by mathematical induction. For if we repeat the substitution once more, putting

$$
x_{1}^{\prime \prime}=\rho x_{1}^{\prime}, \quad x_{2}^{\prime \prime}=x_{1}^{\prime}+\rho x_{2}^{\prime}, \quad \cdots, \quad x_{r}^{\prime \prime}=x_{r-1}^{\prime}+\rho x_{r}^{\prime},
$$

we obtain equations for the $x_{i}^{\prime \prime}$ in terms of the $x_{i}$ which are the same as (3) but with $m+1$ in place of $m$. 
One consequence of the form of (3) is so immediate as to be worthy of note in passing, although not a new result. Suppose $C^{\prime}$ and therefore $C$ to be a periodic substitution in $n$ variables so that $C^{p} \equiv 1$. The first equation of (3) shows that $\rho$ must be a $p$ th root of unity. In the second equation it is impossible for $x_{2}^{\prime}$ and $x_{2}$ to be identical for $m=p>1$. Consequently a necessary condition for a periodic substitution is that all the roots $\rho_{i}$ of the characteristic equation shall be $p$ th roots of unity and that every elementary divisor shall be of first degree. This condition is also obviously sufficient.

$\$ 3$. Introduction of a continuous parameter.-When $m$ is not a positive integer, the component substitutions (3), taken together, still define a substitution which I shall denote by $C^{m}$. To remove the ambiguity lurking in $\rho^{m}$, it shall be understood that $\rho$ is expressed in the form $|\rho| e^{i \vartheta}$ when $2 n \pi \leqq \vartheta \leqq 2(n+1) \pi$ ( $n$ being an integer), and the $m$ th power is formed by the usual rule.

Consider next the effect of performing a substitution $C^{h}$ after $C^{k}$. The resulting substitution is seen at once to be made up of components of the form:

$$
\begin{aligned}
& x_{1}^{\prime \prime}=\rho^{h+k} x_{1}, \\
& x_{2}^{\prime \prime}=c_{21} \rho^{h+k-1} x_{1}+\rho^{h+k} x_{2}, \\
& \cdot \cdot \cdot \cdot \cdot \cdot \cdot \cdot \cdot \cdot \cdot \cdot \\
& x_{r}^{\prime \prime}=c_{r 1} \rho^{h+k-r+1} x_{1}+c_{r 2} \rho^{h+k-r+2} x_{2}+\cdots+c_{r},{ }_{r-1} \rho^{h+k-1} x_{r-1}+\rho^{h+k} x_{r},
\end{aligned}
$$

in which the coefficients $c_{i j}$ are polynomials in $h$ and $k$. Put now $m=h+k$ in (3) and compare the coefficients of (3) and of (4). We know that any two corresponding coefficients are equal to one another for positive integral values of $h$ and $k$. If $h$ only is given a positive integral value, the two coefficients are identical polynomials in $k$, inasmuch as they are equal to one another for an infinity of values of $k$. Consequently corresponding coefficients in these two polynomials in $k$ are equal. But being equal for all positive integral values of $h$, they must be identical polynomials in $h$. We conclude therefore that for $m=h+k$ corresponding coefficients in (3) and (4) are identical. Thus for all values of $h$ and $k$ we have

$$
C^{h+k}=C^{h} C^{k}=C^{k} C^{h} .
$$

In particular, $C^{-h}$ is the inverse of $C^{h}$, since $C^{0} \equiv 1$.

$\S 4$. The $p$-th root of a collineation.-Let $p$ denote henceforth a positive integer. To obtain a linear substitution whose $p$ th power is a given substitution $C^{\prime}$ we have merely to put $m=1 / p$ and take $T^{-1} C^{1 / p} T$. In each component (3) of $C^{1 / p}$ we may choose for $\rho_{i}^{1 / p}$ any one of the $p$ th roots of $\rho_{i}$. Hence if $C$ is made up of $q$ component substitutions, we obtain in this manner $p^{q}$ substitutions whose $p$ th power is the given substitution. 
The question now arises whether all possible roots of a given substitution or collineation are thus obtained. That this is not always the case is apparent on taking the identical collineation $x_{i}^{\prime}=\rho x_{i}(i=1, \cdots, n)$ as the given one. Indeed, we shall now see that whenever $C$ contains two component substitutions haring erfual values of $\rho$, there must be an infinity of distinct $p$ th roots. Suppose $x_{1}, \cdots, x_{r}$ and $y_{1}, \cdots, y_{s}(s \geqq r)$ to be the variables belonging to two such components. Then without destroying the normal form (1) the $r$ variables $x_{i}$ can be replaced by $z_{i} \equiv x_{i}+k y_{i}(i=1, \cdots, r)$, where $k$ denotes an arbitrary constant. Let $C$ be denoted by $C_{z}$ after these $z_{i}$-coördinates have been introduced. Clearly $C_{z}^{1 / p}$ is a $p$ th root* of $C$ which contains the substitutions

$$
z_{1}^{\prime}=\rho^{1 / p_{1}} z_{1}, \quad y_{1}^{\prime}=\rho^{1 / p_{2}} y_{1},
$$

where $\rho^{1 / p_{1}}, \rho^{1 / p_{2}}$ denote $p$ th roots of $\rho$. If different $p$ th roots are here selected, the only planes of the pencil $z_{1}+c y_{1}=0$ which are invariant under the transformation $C^{1 / p}$ are $z_{1}=0$ and $y_{1}=0$. Now the position of $z_{1}=0$ varies with the choice of $k$. Hence by varying its value we obtain an infinity of different $p$ th roots $C^{1 / p}$. It will be shown later that only in the class of cases under consideration will the number of $p$ th roots of $C$, and therefore also of $C^{\prime}$, exceed $p^{q}$. By way of anticipation we may therefore state the following conclusion:

If $q$ is the number of elementary dirisors of (2), the number of $p$-th roots of a given substitution $C^{\prime}$ is cqual to $p^{q}$, unless there are two elementary divisors having equal roots $\rho$, when the number of $p$-th roots will be infinite. $\dagger$

When $C^{\prime}$ is interpreted as a collineation in space of $n-1$ demensions, the number $p^{q}$ is to be replaced by $p^{q-1}$ inasmuch as $p$ substitutions correspond to one collineation owing to the homogeneous character of the variables.

This result is, however, only incidental. Our concern here is not with the number of $p$ th roots but rather with the form into which $C$ and any one of its $p$ th roots can be simultaneously thrown. I shall show that by properly choosing the coördinate planes of reference any particular root under consideration may be put into the form $C^{1 / p}$ without destroying the normal form of $C$. For brevity of statement it will be said that any substitution (collineation) has the normal form (1) if euch component substitution (collineation) is of this form.

Denote by $K$ any particular $p$ th root of $C$ under consideration, and suppose first that $K$ itself has first been thrown into normal form (1). Then $C$ as its $\eta$ th iterative has the form (3) where $m=p$. Make now simultaneously in

${ }^{*} C^{\mathrm{i} / \mu}$ is uscd throughout to denote, not any collineation whose $p$ th power is $C$, but one which is obtained by putting $m=1 / p$ in each component (3) of $C^{m}$.

$\dagger$ The $p$ th roots of a given matrix or substitution have been considered in a very different marner by Sylvester; Comptes Rend u s, vol. 94 (1882), pp. 55, 396. Sylvester's results for the number of $p$ th roots is not complete. He obtains the number only for the simple case $q=n$, and for a certain class of cases for which the number is infinite.

Trans. Ain. Math. Soc. 23 
each component of $K$ and $C$ a change of variable of the form:

$$
\begin{aligned}
& x_{1}=y_{1}, \\
& x_{2}=y_{1}+c_{22} y_{2}, \\
& x_{3}=y_{1}+c_{32} y_{2}+c_{33} y_{3}, \\
& \cdot \cdot \cdot \cdot \cdot \cdot \cdot \\
& x_{r}=y_{1}+c_{r 2} y_{2}+\cdots+c_{r r} y_{r},
\end{aligned}
$$

and determine the $c_{i j}$ so that the component of $C$ shall be transformed into normal form:

$$
\begin{aligned}
& y_{1}^{\prime}=\rho^{p} y^{\prime}, \\
& y_{i}^{\prime}=y_{i-1}+\rho^{p} y_{i} \quad(i=2,3, \cdots, r) .
\end{aligned}
$$

We shall see first that the $c_{i j}$ are thereby uniquely determined. For this purpose let the equations (3) for $C$ (wherein $m=p$ ) be abridged by

$$
x_{i}^{\prime}=d_{i 1} x_{1}+d_{i 2} x_{2}+\cdots+d_{i, i-1} x_{i-1}+\rho^{p} x_{i} \quad(i=1, \cdots, r) .
$$

Then by the change of variables $(6)$ they become

$$
\begin{aligned}
c_{i i} y_{i}^{\prime}+c_{i, i-1} & y_{i-1}^{\prime}+\cdots+c_{i 2} y_{2}^{\prime}+y_{1}^{\prime} \\
& =\sum_{j=1}^{i-1} d_{i j}\left(y_{1}+c_{j 2} y_{2}+\cdots+c_{j j} y_{j}\right)+\rho^{p}\left(y_{1}+\cdots+c_{i i} y_{i}\right) .
\end{aligned}
$$

If, now, all the $c_{j k}(j \leqq i-1)$ have been determined so that the first $i-1$ of equations (7) are fulfilled, the equations just written become in consequence

$$
c_{i i} y_{i}^{\prime}=\sum_{j=1}^{i-1} d_{i j}\left(y_{1}+c_{j 2} y_{2}+\cdots+c_{j j} y_{j}\right)+\rho^{p} c_{i i} y_{i}-\sum_{j=2}^{i-1} c_{i j} y_{j-1} .
$$

To make this equation identical with the $i$ th relation of (7) we must put:

$$
\begin{array}{rlrl}
c_{i 2} & =\sum_{j=1}^{i-1} d_{i j}, \\
c_{i k} & =\sum_{j=k-1}^{i-1} d_{i j} c_{j, k-1} & (k=3, \cdots, i-1), \\
c_{i i} & =d_{i, i-1} c_{i-1, i-1} \neq 0 & \left(c_{11}=1\right) .
\end{array}
$$

These equations therefore determine uniquely $c_{i 2}, c_{i 3}, \cdots, c_{i i} \neq 0$, after the $c_{i-1, j} c_{i-2, j}, \cdots$ have been already similarly determined. Thus the coefficients of (6) are uniquely determined by the requirement the $C$ shall be transformed into normal form ( 7 ), as was to be proved.

It remains to ascertain what $K$ will simultaneously become in consequence of the transformation. Since by hypothesis $K$ was initially in normal form 
(1), the change of variables (6) throws its components into the form:

$$
\begin{aligned}
& y_{1}^{\prime}=\rho y_{1}, \\
& y_{i}^{\prime}=b_{i 1} y_{1}+\cdots+b_{i, i-1} y_{i-1}+\rho y_{i} \quad(i=2, \cdots, r),
\end{aligned}
$$

where the coefficients $b_{i j}$ are yet to be ascertained. Obviously the $p$ th iterative of (8) has the same form as (8) itself, $\rho$ being replaced by $\rho^{p}$ and the other coefficients by polynomials in terms of the $b_{i j}$. We know further that the $p$ th iterative $C$ has the form ( $\bar{\tau}$ ). If now in these two expressions for this iterative we equate corresponding coefficients, it will be found that we obtain a set of equations of the following character:

$$
\begin{aligned}
p b_{21} \rho^{p-1} & =1 ; \\
p b_{32} \rho^{p-1} & =1, \\
p b_{31} \rho^{p-1}+[\quad] & =0 ; \\
p b_{43} \rho^{p-1}+[\quad] & =1, \\
p b_{42} \rho^{p-1}+[\quad] & =0, \\
p b_{41} \rho^{p-1}+[\quad] & =0,
\end{aligned}
$$

where in each line the bracket stands for a polynomial in the $b_{i j}$ of the previous lines. Hence the $b_{i j}$ are uniquely determined, and consequently there is but a single substitution (8), with given $\rho$, whose $p$ th power is (7). Now (7) is the same as (1) with $\rho^{p}$ in place of $\rho$. Consequently if we replace $\rho$ by $\rho^{p}$ in (3), put $m=1 / p$, and then substitute $y_{i}, y_{i}^{\prime}$ for $x_{i}, x_{i}^{\prime}$, we shall obtain a $p$ th root of form (8) with the given $\rho$. As this root was obtained from (3) by placing $m=1 / p$, the statement has been thus finally verified that $C$ can be so transformed into normal form (1) that any particular one of its p-th roots shall be simultaneously of form $C^{1 / p}$.

§5. The derivation of the one parameter collineation group.-From equation (5) it is evident that we obtain a continuous one-parameter group $C^{m}$ by varying $m$ continuously through real values in (3). For brevity it will be said that any group has the normal form (3) when it consists of components of this form. The question next to be considered is whether every continuous one-parameter collineation group can be thrown into this normal form.

In the consideration of this question it will be assumed that if any substitution is contained in the group, its inverse and one of its square roots are also contained therein. Thus with any particular substitution $C$, assumed in normal form, will be included a succession of $2^{N}$-th roots $(N=1,2, \cdots)$. Our aim is to establish next that this entire succession of roots may be simul- 
taneously thrown into the form $C^{m}\left(m=1 / 2^{N}\right)$ by proper choice of the coördinate planes.

To this end consider first what change of variables

$$
y_{i}=k_{i 1} x_{1}+\cdots+k_{i n} x_{n} \quad(i=1, \cdots, n)
$$

can be made without destroying the normal form of $C$. In terms of the $y_{i}$ the substitution $C$ is to consist of exactly the same number of components with the same orders as in terms of the $x_{i}$. Take a sample component

$$
y_{1}^{\prime}=\rho^{\prime} y_{1}, \quad y_{i}^{\prime}=y_{i-1}+\rho^{\prime} y_{i} \quad(i=2, \cdots, s),
$$

in which to make the substitution (9). Since the $x$-variables were separated in $C$ into independent sets (1), our equations (10) after the change of variables (9) may be split up into sets of equations, each involving only the $x$-variables of a single component collineation. Thus corresponding to (1) we obtain the $s$ equations:

$$
\begin{aligned}
& k_{11} x_{1}^{\prime}+\cdots+k_{1 r} x_{r}^{\prime}=\rho^{\prime}\left(k_{11} x_{1}+\cdots+k_{1 r} x_{r}\right), \\
& k_{i 1} x_{1}^{\prime}+\cdots+k_{i r} x_{r}^{\prime}=\rho^{\prime}\left(k_{i 1} x_{1}+\cdots+k_{i r} x_{r}\right) \\
& \quad+\left(k_{i-1,1} x_{1}+\cdots+k_{i-1, r} x_{r}\right) \quad(i=2, \cdots, s) .
\end{aligned}
$$

If the values for $x^{\prime}$ given in (1) are now substituted, these equations become identities. Unless $\rho^{\prime}=\rho$, we see that in the first of these identities every $k_{1 i}=0$, and then from the succeeding ones that every $k_{i j}$ vanishes. In other words, $y_{1}, \cdots, y_{s}$ can involve only the $x$-variables of component substitutions which have the same value of $\rho$. When $\rho=\rho^{\prime}$, the identities give

$$
\begin{array}{r}
0=k_{12}=k_{13}=\cdots=k_{1 r}, \quad k_{i-1, r}=0, \quad k_{i j}=k_{i-1, j-1} \\
(i=2, \cdots, s ; j=2, \cdots, r) .
\end{array}
$$

Hence the part of $y_{1}, \cdots, y_{s}$ which involves $x_{1}, \cdots, x_{r}$ is as follows:

$$
\begin{aligned}
& \bar{y}_{1}=k_{s s} x_{1} \text {, } \\
& \bar{y}_{2}=k_{s, s-1} x_{1}+k_{s s} x_{2} \text {, } \\
& \bar{y}_{s-1}=k_{s 2} x_{1}+k_{s 3} x_{2}+\cdots+k_{s s} x_{s-1} \text {, } \\
& y_{s}=k_{s 11} x_{1}+k_{s 2} x_{2}+\cdots+k_{s, s-1} x_{s-1}+k_{s 8} x_{s} . \\
& \left(k_{s i}=0 \text { if } s>r\right) \text {. }
\end{aligned}
$$

The change of variables (9) therefore consists of components

$$
y_{i}=\Sigma \bar{y}_{i} \quad(i=1, \cdots, s),
$$

where the $y_{i}$ summed are expressions (11), each of which contains the $x_{i}$ of a single component (1) having $\rho=\rho^{\prime}$.

We proceed next to consider the effect of this change of variables upon 
$C^{1 / p}$. Suppose that $C$ comprises $q$ components, corresponding to the values

$$
\rho_{1}, \rho_{2}, \cdots, \rho_{q} .
$$

Then the corresponding sequence of roots for $C^{1 / p}$ is

$$
\rho_{1}^{1 / p}, \rho_{2}^{1 / p}, \cdots, \rho_{q}^{1 / p} .
$$

In case any two members $\rho_{i}, \rho_{j}$ of (12) are equal, we shall take the corresponding roots in (13) equal. Then it will be shown quickly that our change of variables which left $C$ unaltered in form must also leave the form of this $C^{1 / p}$ unaltered. First, it is clear that any change of variable (11) leaves the first $s$ equations of (3) unaltered in form, for if we multiplied the first $l$ equations of (3) successively by $k_{s, s-l+1}, k_{s, s-l+2}, \cdots, k_{s s}$ and add, we obtain again the $l$ th equation of (3) with $\bar{y}_{i}^{\prime}, \bar{y}_{i}$ in place of $x_{i}^{\prime}, x_{i}$. Secondly, if we have several sets of variables $\bar{y}_{i}^{\prime}, \bar{y}_{i}$ satisf ying the same set of equations of form (3), then the sums of corresponding variables $\Sigma \bar{y}_{i}^{\prime}, \Sigma \bar{y}_{i}$ also satisfy such a set of equations. It follows therefore that we have exactly the same equations for $C^{1 / p}$ in terms of the $y_{i}$ as in terms of the $x_{i}$. Accordingly the form of $C^{1 / p}$ is unaltered.

Consider next the totality of $p$ th roots of $C(p=1,2, \cdots)$, which fulfill the condition that any two members of the sequence (13) are equal when the corresponding members of (12) are equal. We have found above that without destroying the normal form of $C$ any one of these roots may be brought into the normal form $C^{1 / p}$, and we have just proved that then any change of variable which does not affect the form of $C$ will also not destroy the normal form $C^{1 / p}$, provided the sequences (12) and (13) are alike in respect to the equality or inequality of their terms. It follows that the entire set of roots now under consideration will simultaneously assume the normal form $C^{1 / p}$ whenever $C$ itself has the normal form (1). In particular, if no two members of the sequence (12) are equal, every $p$ th root $(p=1,2, \cdots)$ will have the form $C^{1 / p}$. Now for a fixed value of $p$ the number of roots of this form is $p^{q}$, since there are in all $q$ components (3) and in each we have a choice of $p$ values for $\rho^{1 / p}$. Thus we have established the conclusion italicized in $\S 4$, to the effect that when no two members of (12) are equal, the number of $p$ th roots of $C$ is exactly $p^{q}$.

We are now ready to proceed with the derivation of the normal form of a continuous one-parameter collineation group. With a given collineation $C$, assumed in normal form, there is also included in the group a succession of $2^{n}$ th roots $(n=1,2, \cdots)$ of $C$. Since each of these is a square root of the preceding, each $\rho_{i}{ }^{1 / 2^{n+1}}$ in (13) is a square root of the corresponding $\rho_{i}^{1 / 2^{n}}$. Hence as $n$ increases indefinitely, we reach a point subsequent to which any two members of (13), if still equal, henceforth remain so. Suppose the permanent status to be reached for $n \geqq m$. Then let $K$ denote the particular $2^{m}$ th 
root of $C$ which is included in our succession of its roots. When $K$ is thrown into normal form (1), all the succeeding roots by the last paragraph must have the form $K^{1 / p}$ where $p=2^{n-m}$. Also $C$ as the $m$ th iterative of $K$ has the form $K^{m}$. Suppose now that $C$ by a change of variable, as explained in $\S 4$, is brought back into normal form (1). Then $K$ takes the form $C^{1 / 2 m}$ and its $i$ th powers the form $C^{i / 2 m}$. Its successive $p$ th roots $\left(p=2^{n-m}\right)$ before the change of variable (6) had the form (8), with $\rho^{1 / 2^{n}}$ in place of $\rho$, and they remain of the same form after the transformation. But in $\$ 4$ we saw that there was but a single substitution of such form, with preassigned values of $\rho_{i}^{1 / 2^{n}}$, whose $2^{n}$ th power was $C$, and that substitution had the form $C^{1 / 2^{n}}$. It follows that our succession of $2^{n}$ th roots of $C(n=1,2, \cdots)$ by proper choice of the coordinate system may all be put simultaneously into the standard form $C^{1 / 2 n}$.

We now have in our group all substitutions of the form $C^{1 / 2^{n}}$ and with them their $p$ th iteratives $C^{p / 2^{n}}$ and their inverse substitutions $C^{-p / 2^{n}}$. Thus our group has been found to contain substitutions of form $C^{m}$ for a dense set of values of $m$, namely $m= \pm p / 2^{n}(p, n=1,2, \cdots)$. It remains to extend this conclusion to all real values of $m$. By hypothesis the group is continuous. Hence in the first equation of (3),

$$
x_{1}^{\prime}=\rho^{m} x_{1}
$$

such a succession of roots $\rho^{m}$ must be selected for $m=p / 2^{n}$ as will yield a continuous function. Obviously a unique continuous modulus $|\rho|^{m}$ is thereby determined. Let $f(m)$ denote the real part of $e^{i \arg \rho^{m}}$. From equation (5) it follows that for values of $h$ and $k$ selected from the dense set $\left\{ \pm p / 2^{n}\right\}$ $f(m)$ must satisfy the cosine law, and accordingly

$$
f(h+k)-f(h-k)=2 f(h) f(k) .
$$

Since also $f(m)$ is continuous, this equation must consequently subsist for all real values of $h$ and $k$. But the real and continuous functions $f(x)$ which satisfy this equation and do not exceed 1 in absolute value were shown by Cauchy to be $f(x)=0,1, \cos c x$. Consequently for real values of $m$ the real part of $e^{i \arg \rho^{m}}$ is $\cos (m \arg \rho)$, and hence the group contains the substitution $x_{1}^{\prime}=\rho^{m} x_{1}$.

In every equation of (3) subsequent to the first, $\rho^{m}$ appears in the coefficients multiplied by a polynomial in $m$ with real coefficients. As the equations subsist for the dense set of values $m= \pm p / 2^{n}$, it follows from considerations of continuity that they must also hold for all real values of $m$. We thus reach the following conclusion:

Every continuous one-parameter collineation group in $n$ homogeneous variables with real parameter $m$, or homogeneous one-parameter linear substitution group in $n$ variables, may be thrown by proper choice of coördinates into a normal form consisting of components of the form (3). 
For $m=0$ the equations (3) give the identical transformation. The infinitesimal transformation which generates the group may be obtained by assuming $m$ to be infinitesimal. For convenience put $m=d t$, then on expanding the coefficients of (3) in ascending powers of $d t$ and neglecting all but the first power of $d t$ we obtain the increments*

$$
\begin{array}{r}
d x_{i}=x_{i}^{\prime}-x_{i}=d t\left[\frac{(-1)^{i} x_{1}}{(i-1) \rho^{i-1}}+\frac{(-1) \stackrel{i-1}{\cdot} x_{2}}{(i-2) \rho^{i-2}}+\cdots+\frac{x_{i-1}}{\rho}+x_{i} \log \rho\right] \\
(i=1, \cdots, r) .
\end{array}
$$

The reality of the infinitesimal transformation will be discussed later.

\$6. Lists of one-parameter collineation groups.-Our conclusion concerning the form of continuous one-parameter collineation groups may be applied at once to the construction of a complete list of such groups for space of $n-1$ dimensions. For this purpose we write down first in all possible ways sets of component collineations of the form (3) for which the sum of the orders $r$ will be equal to $n$. Then under each set of $n$ equations so constructed we are to distinguish all possible cases which may arise through the equality or nonequality of the $\rho_{i}$ of the component collineations, inasmuch as these cases differ from one another in respect to the character of the invariantive configuration of points, lines, planes, etc. Thus for $n=3$ we obtain in addition to the identical transformation the following types of one-parameter collineation groups $(m=$ parameter $)$ :

Type $I$.

$$
\begin{aligned}
& x_{1}^{\prime}=\rho_{1}^{m} x_{1}, \\
& x_{2}^{\prime}=\rho_{2}^{m} x_{2}, \\
& x_{3}^{\prime}=\rho_{3}^{m} x_{3}
\end{aligned}
$$

$$
\left(\rho_{1} \neq \rho_{2} \neq \rho_{3}\right) .
$$

Type II. $\quad x_{1}^{\prime}=\rho_{1}^{m} x_{1}$,

$$
\begin{aligned}
& x_{2}^{\prime}=m \rho_{1}^{m-1} x_{1}+\rho_{1}^{m} x_{2}, \\
& x_{3}^{\prime}=\rho_{3}^{m} x_{3}
\end{aligned}
$$

Type III.

$$
\begin{aligned}
x_{1}^{\prime} & =\rho^{m} x_{1}, \\
x_{2}^{\prime} & =m \rho^{m-1} x_{1}+\rho^{m} x_{2}, \\
x_{3}^{\prime} & =\frac{m(m-1)}{2 !} \rho^{m} x_{1}+m \rho^{m-1} x_{2}+\rho^{m} x_{3} .
\end{aligned}
$$

Type IV. Same as I but with $\rho_{2}=\rho_{3}$.

Type V. Same as II but with $\rho_{1}=\rho_{3}$.

* Taking all such sets of increments we obtain a canonical form for the symbol of an infinitesimal collineation more explicit than that given in LIE-ENGEL's Theorie der Transformationsgruppen, vol. 1, top of p. 585 . 
These five types of groups correspond to the familiar five varieties of plane collineations which result on placing $m=1$. In the literature they occur mostly in unhomogeneous form. One such form results on dividing the last two of our three equations by the first and is as follows:*

$$
\text { ( } a=\text { parameter, } \quad k=\text { constant } \text { ) }
$$

I. $x^{\prime}=a x$,

$y^{\prime}=a^{k} y(k \neq 1)$.

II. $x^{\prime}=a+x$,

$y^{\prime}=k^{a} y(k \neq 1)$.
IV. $x^{\prime}=a x$, $y^{\prime}=a y$.

V. $x^{\prime}=x+a$,

$y^{\prime}=y$.

III. $x^{\prime}=x+a$,

$y^{\prime}=y+a x+\frac{a(a-k)}{2}$.

A corresponding enumeration of the 13 types of one-parameter collineation groups in three dimensions will be found in the course of $\$ 12$. While such an enumeration for $S_{3}$ has been given previously; $\dagger$ it is to be emphasized that the advantage of the present results is that they can be applied with equal ease and directness to a like enumeration of one-parameter collineation groups in finite form for n-dimensional space. So far as I know, this has not been previously attained.

\$7. Homogeneous one-parameter groups in $n$ variables.-A second application of the result of $\S 5$ is the construction of list of homogeneous linear groups in $n$ variables. In this case certain distinctions must be considered, which do not arise in the case of homogeneous coördinates and which are due to the interpretation of the one-parameter group as an affine group in $S_{n}$, whereby the plane at infinity is left unaltered. These distinctions are perceived on bringing in an $(n+1)$-th variable to render the coördinates homogeneous. This merely adds to our system another equation, $x_{n+1}^{\prime}=\rho_{n+1} x_{n+1}$, in which $\rho_{n+1}=1$. Hence the character of the complete invariantive configuration of the group is not merely contingent upon the system of equalities and inequalities which subsist between the $\rho_{i}$ of the component collineations, but it also depends upon their equality or non-equality to the special value $\rho_{n+1}=1$. A second point of difference is that the collineation $x_{i}^{\prime}=\rho x_{i}(i=1, \cdots, n)$ is no longer to be neglected, since it no longer furnishes the identical transform-

* Cf. Fr. MEyer, Mathematical papers read at the International Mathematical Congress, Chicago, 1893, pp. 189-190. The above forms can be obtained by integrating the five types of plane infinitesimal collineations given by Lie-Scheffers, p. 65. In type III the term - ak / 2 can be removed by change of variable $x=\bar{x}+k / 2$.

† Given in unhomogeneous form by Newson, Kansas University Quarterly, July, 1898, pp. 140-141. 
ation. As a specimen application I shall enumerate the homogeneous oneparameter groups for $n=3$.

Types $\mathrm{I}-\mathrm{V}$ are the same as the types $\mathrm{I}-\mathrm{V}$ enumerated in $\$ 6$ but with the added restriction that each $\rho_{i} \neq 1$. We have also in addition

$$
\text { Type VI. } \quad x_{i}^{\prime}=\rho^{m} x_{i} \quad(\rho \neq 1 ; i=1,2,3) .
$$

The remaining types are obtained from these by placing one or more of the $\rho_{i}$ equal to 1 . We may neglect all cases in which one coördinate remains unaltered, provided the changes in the other two are independent of that coördinate, since then the group is essentially a group in two variables. There remain the two following cases.

Type VII. Same as II but with $\rho_{1}=1$.

$$
x_{1}^{\prime}=x_{1}, \quad x_{2}^{\prime}=m x_{1}+x_{2}, \quad x_{3}^{\prime}=\rho_{3}^{m} x_{3} \quad\left(\rho_{3} \neq 1\right) .
$$

Type VIII. Same as III but with $\rho=1$.

$$
\begin{aligned}
x_{1}^{\prime} & =x_{1}, \\
x_{2}^{\prime} & =m x_{1}+x_{2}, \\
x_{3}^{\prime} & =\frac{m(m-1)}{2 !} x_{1}+m x_{2}+x_{3} .
\end{aligned}
$$

The simplicity with which this enumeration is made may be compared with the determination of a complete list of these groups in terms of the infinitesimal transformation; ${ }^{*}$ whence they may be obtained in finite form + by integration.

\$ 8. Real one-parameter groups.-When $m$ traverses its axis of reals, the point $\left(x_{1}^{\prime}, \cdots, x_{n}^{\prime}\right)$ describes a path curve. The group is called real, when every real point describes a real path curve. In other words, it is one which can be generated by a real infinitesimal transformation.

We have now to derive the necessary and sufficient conditions for the reality of the group corresponding to a real initial collineation $C^{\prime}$. By the nature of our derivation of the group the question of its reality resolves itself into two parts. We have first to consider the reality of the reduction of $C^{\prime}$ to normal form, and after this the reality of the group in which $C$ is contained. In the reduction we shall regard the transformation of $C^{\prime}$ through $T$ as a change of variable.

When $C^{\prime}$ is real, the characteristic equation (2) is real and its elementary divisors, so far as they are imaginary, occur only in conjugate pairs of equal degree, $\left(\rho-\rho_{i}\right)^{r}$ and $\left(\rho-\bar{\rho}_{i}\right)^{r}$. It is fairly obvious, and can indeed be strictly verified, that the reduction of $C^{\prime}$ to normal form (1) can be made in

* Lie-Enger, loc. cit., vol. 3, p. 119, or Lie-Scheffers, loc. cit., p. 522.

† Cf. Fr. Meyer, loc. cit., p. 197-198. 
such a way that to real elementary divisors will correspond real component substitutions (1), while to each conjugate pair of unreal elementary divisors will correspond two components (1) of equal order in which corresponding variables $x_{i}, \bar{x}_{i}$ of the two components are conjugate imaginaries. The variables of the real components (1) are real linear forms in terms of the initial variables of $C^{\prime}$, while the variables $x_{i}, x_{i}$ of the conjugate components are linear forms with conjugate imaginary coefficients. We shall suppose henceforth that the reduction of $C^{\prime}$ to normal form $C$ has been made in such a manner. Then $C$ is not intrinsically imaginary, and to convert it into real form we have merely to place

$$
x_{i}=y_{i}+i z_{i}, \quad \rho=|\rho| \cdot[\cos \vartheta+i \sin \vartheta]
$$

in one of each two conjugate components of (1), and then to split up the equations of (1) into their real and imaginary parts. When the same change is effected in (3), we obtain in place of a pair of conjugate imaginary components of order $r$ the following equivalent set of $2 r$ real equations:

$$
\begin{aligned}
& y_{1}^{\prime}=|\rho|^{m} \cdot\left[y_{1} \cos m \vartheta-z_{1} \sin m \vartheta\right] \text {, } \\
& z_{1}^{\prime}=\left|\boldsymbol{\rho}^{m}\right| \cdot\left[y_{1} \sin m \vartheta+z_{1} \cos m \vartheta\right] \text {, } \\
& y_{i}^{\prime}=\frac{m(m-1) \cdots(m-i+1)}{(i-1) !}|\rho|^{m-i+1} \cdot\left[y_{1} \cos (m-i+1) \vartheta\right. \\
& \left.-z_{1} \sin (m-i+1) \vartheta\right] \\
& +\frac{m(m-1) \cdots(m-i+2)}{(i-2 !)}|\rho|^{m-i+2} \cdot\left[y_{2} \cos (m-i+2) \vartheta\right. \\
& \left.-z_{2} \sin (m-i+2) \vartheta\right]+\cdots+|\rho|^{m} \cdot\left[y_{i} \cos m \vartheta-z_{i} \sin m \vartheta\right] \\
& z_{i}^{\prime}=\frac{m(m-1) \cdots(m-i+1)}{(i-1) !}|\rho|^{m-i+1} \cdot\left[y_{1} \sin (m-i+1) \vartheta\right. \\
& \left.+z_{1} \cos (m-i+1) \vartheta\right]+\cdots+|\rho|^{m} \cdot\left[y_{i} \sin m \vartheta+z_{i} \cos m \vartheta\right] \text {, } \\
& (i=2, \cdots, r) \text {. }
\end{aligned}
$$

These equations give in normal real form the component substitutions which correspond to a pair of conjugate elementary divisors of (2). By placing $m=1$ we obtain the corresponding components of $C^{*}$ itself.

We have thus ascertained the normal form into which a continuous oneparameter group containing a real substitution $C^{\prime}$ can be transformed by real change of variable. It consists, namely, only of components of the form (3) with real $\rho$ and of components of the form (14). We have now to examine their reality when the parameter $m$ is varied continuously through real values. The components (14) are plainly real. On the other hand, (3) will contain 
an imaginary factor $\rho^{m}$ if $\rho$ is negative. In case, however, of the existence of a pair of equal elementary divisors of (2) with real $\rho_{i}$, we can regard them, if we please, as a pair of conjugate imaginary divisors $\left(\rho-\rho_{i}\right)^{r},\left(\rho-\bar{\rho}_{i}\right)^{r}$ in which the common argument of $\rho_{i}, \bar{\rho}_{i}$ is $2 \pi$ or $\pi$ according as $\rho_{i}$ is positive or negative. We may therefore employ, instead of two like components (3) of order $r$, a single sy'stem of $2 r$ equations of form (14). If $\rho_{i}$ is negative, we avoid thereby the introduction of two imaginary components into our group, while if $\rho_{i}$ is positive, we have a choice between two different modes, both real, of generating the pair of like components of $C$. The following conclusion now ensues:

$A$ necessary and sufficient condition that a real substitution $C^{\prime}$ can be included in a real, continuous and homogeneous one-parameter substitution group is that the negative roots of the characteristic equation (2) shall occur in equal pairs of equal order. If there exist one or more pairs of equal positive roots of the same order, $C^{\prime}$ can be included in two or more real substitution groups of different nature.*

The reason for the failure of a real substitution $C^{\prime}$ to belong to a continuous real one-parameter group is to be sought in the inclusion of projective reflections in its make up. By a reflection on the axis or plane of $x_{i}$ is to be understood a transformation $x_{i}^{\prime}=-x_{i}$ which alters the sign of this coördinate, leaving the others unaltered. Suppose now that $\rho$ in (1) is negative and set $\rho=-\rho^{\prime}$. If we then make a change of coördinates by replacing each $x_{i}, x_{i}^{\prime}$ of even subscript by its negative, equations (1) become

$$
\begin{aligned}
& -x_{1}^{\prime}=\rho^{\prime} x_{1}, \\
& -x_{i}^{\prime}=x_{i-1}+\rho^{\prime} x_{i} \quad(i=2, \cdots, r),
\end{aligned}
$$

Clearly this transformation can be resolved into two which are commutative, the one being

$$
\begin{aligned}
& x_{1}^{\prime}=\rho^{\prime} x_{1} \\
& x_{i}^{\prime}=x_{i-1}+\rho^{\prime} x_{i} \quad(i=2, \cdots, r) .
\end{aligned}
$$

while the other consists of $r$ reflections changing the signs of $x_{1}^{\prime}, \cdots, x_{r}^{\prime}$. Now it is well known that an even but not an odd number of reflections can be generated by a real infinitesimal projective transformation. In fact, an even number of reflections $y_{i}^{\prime}=-y_{i}, z_{i}=-z_{i}(i=1, \cdots, r)$ is included in a continuous group

$$
\begin{aligned}
& y_{i}^{\prime}=y_{i} \cos m \pi-z_{i} \sin m \pi, \\
& z_{i}^{\prime}=y_{i} \sin m \pi+z_{i} \cos m \pi
\end{aligned} \quad(i=1, \cdots, r),
$$

*I. e., real groups differing in respect to the number or order of their components (3) and (14). If in constructing (14) the arguments of $\rho, \bar{\rho}$ are changed by $2 \pi$ and $-2 \pi$ respectively, this changes the value of $\vartheta$ in (14) by $2 \pi$ but does not change essentially the nature of the group. 
which is commutative with (14). But we have found that a pair of components (15) of equal order and with equal $\rho^{\prime}$ can be included in a group (14) if we there put $\vartheta=2 \pi,|\rho|=\rho^{\prime}$ and take $y_{i}, z_{i}$ to be the $r$ variables of the two components (15). As (14) and (16) are commutative, we see thus why the condition that the negative $\rho_{i}$ shall occur only in equal pairs of equal orders is sufficient to insure the inclusion of $C^{\prime}$ in a real continuous group. We also saw above that this condition is necessary.

Consider next the reality of the group containing $C^{\prime}$ when $C^{\prime}$ is interpreted, not as a homogeneous linear substitution, but as a collineation in $n$ homogeneous variables. A slight modification in the above results is now needed owing to the fact that we can suppress or supply a common factor in all the $n$ variables $x_{i}^{\prime}$ of the collineation. The difficulty before arose from an imaginary factor $\rho^{m}$ in the right-hand members of (3) when $\rho$ was negative. But we are now at liberty to introduce a common factor $(-1)^{m}$ into all the $n$ variables $x_{i}^{\prime}$. If at the same time in each of the components (3) a change of coördinates is made by replacing every $x_{i}^{\prime}, x_{i}$ of even subscript by its negative, we obtain the same equations as at start save for a change in the sign of every $\rho$. Thus the rôles of the positive and the negative roots of the characteristic equation are interchanged, while the argument of each imaginary root is changed by $\pi$. We reach therefore the following criterion for the reality of our group:

The necessary and sufficient condition that a real collineation $C^{\prime}$ in $n$ homogeneous variables can be included in a continuous and real one-parameter collineation group or, in other words, that it can be generated by a real infinitesimal collineation, is that either the elementary divisors which correspond to negative roots of (2) or those which correspond to positive roots shall occur only in equal pairs, or not at all.*

If this condition holds for the positive (negative) roots and there is at least one pair of equal negative (positive) roots of equal order, then $C^{\prime}$ can be included in two or more real groups of different nature, and only then.

The conclusion concerning the form of the real group which has been reached, though not expressly stated, can now be summed up as follows:

Every real and continuous one-parameter collineation group in $n$ homogeneous variables, or homogeneous group in $n$ variables, by proper choice of the coördinate planes can be expressed in a normal form which consists only of components of form (3), in which the $\rho_{i}$ are positive, and of components of form (14).

$\S 9$. Lists of real one-parameter groups.-The method of $\$ \S 6,7$ can be extended to construct lists of linear and homogeneous one-parameter groups under the added requirement that the groups shall be real. We have first

* This condition has been previously obtained by TABER who gave the condition in terms of the numbers of Sylvester. Cf. Bulletin of the American Mathematical Societ $y$, vol. 2 (1896), pp. 225-7, theorems 1,2 and 6 . The connection between these numbers and the degree of the elementary divisors is explained by him in vol. 3, pp. 157-8. 
to throw together in all different ways sets of equations of form (3) and (14) for which the total number of equations shall be equal to $n$, and in those of form (3) $\rho$ is now restricted to be positive. Then for each different combination of $n$ equations all possible cases are to be distinguished which differ in respect to the invariantive configuration of points, lines, planes, etc., due account being taken of the homogeneous or non-homogeneous nature of the coördinates. For $n=3$ we get seven types of real one-parameter collineation groups. Five of these are the same as in $\S 6$, except that the $\rho_{i}$ are now restricted to be positive. In addition, we have the two following types:

Type VI. $\quad x_{1}^{\prime}=\rho_{1}^{m} x_{1}$,

$$
\begin{aligned}
& y^{\prime}=\left|\rho_{2}\right|^{m} \cdot[y \cos m \vartheta-z \sin m \vartheta], \\
& z^{\prime}=\left|\rho_{2}\right|^{m} \cdot[y \sin m \vartheta+z \cos m \vartheta] \quad\left(\rho_{2}=\left|\rho_{2}\right| e^{i \vartheta}\right),
\end{aligned}
$$

in which $\rho_{1} \neq\left|\rho_{2}\right|$.

Type VII. The same but with $\rho_{1}=\left|\rho_{2}\right| .^{*}$

A list for $n=4$ is scattered through $\S 12$, the total number of real types distinguished being 19. No account is there taken of distinctions similar to that just made between types VI and VII.

Of real homogeneous groups in three variables there are eleven types, eight of which have been already given in $\S 7$, but the $\rho_{i}$ therein are now taken to be positive. In addition, we have the types VI and VII just given with the added restriction $\rho_{1} \neq 1,\left|\rho_{2}\right| \neq 1$, and an eleventh type which is the same as VI above but $\left|\rho_{2}\right|=1,\left|\rho_{1}\right| \neq 1$. When $\rho_{1}=1$ in VI, the group is essentially one in two variables.

Part II. The Classification of Collineations. Space $W$-curves.

\$10. The mode of classification.-The principle to be used for the classification of collineations is that of infinite iteration. As stated in the introduction, we assume at start the usual classification given by the theory of elementary divisors. The standard form there obtained for a collineation depends upon the nature of the system of elementary divisors of its characteristic equation, or, geometrically considered, upon the character of the invariantive configuration of points, lines, planes, etc. The equalities and inequalities subsisting between the roots $\rho_{i}$ of the elementary divisors are thereby involved. When collineations of the same standard type are iterated, differences arise which depend upon the relative magnitude of the moduli of the $\rho_{i}$. Hence we get a further subdivision into classes. Although the effects of the iteration of a collineation and its inverse may be very different, it will be convenient never-

* For these groups in terms of the infinitesimal transformation see LiE-Schefrers, loc. cit., pp. 74-82. 
theless to include both under the same class, inasmuch as they belong to a common group. Thereby the number of different classes is somewhat diminished.

The iterative principle applies alike to real and unreal collineations, demanding nothing beyond a knowledge of the characteristic equation. To effect the iteration of $C$ we increase $m$ through positive integral values in each component of $C^{m}$, while to iterate the inverse collineation $m$ is to be decreased through negative integers. Instead, however, of changing $m$ discontinuously we might allow it to increase or decrease continuously through real values. Thus our classification is also simultaneously one for continuous one-parameter collineation groups. It is, however, to be observed that if account is taken of reality, there is a very restricted class of cases (cf. $\$ 8$, near end) in which a real collineation can be included in two or more real collineation groups of different nature. To this extent the parallelism between the classification of collineations and of one-parameter collineation groups fails.

When $m$ traverses its axis of reals starting from $m=0$, the point $P^{\prime}=\left(x_{1}^{\prime}, \cdots, x_{n}^{\prime}\right)$ starts from an initial position $P=\left(x_{1}, \cdots, x_{n}\right)$ and describes a path curve real or imaginary. These curves are sometimes called $W$-curves on account of their projective properties and are of especial interest when real. With the accompanying group they are then generated by real infinitesimal transformation. Thus our classification for real groups becomes one also for the system of path curves and is based essentially upon the character of this system.

An interesting extension of the notion of a $\mathrm{W}$-curve will be obtained by allowing $m$ to roam over its imaginary domain. Then the system of the components (3) and (14) of $C^{m}$ is to be interpreted in a real space of $2 n$-dimensions, in which $P^{\prime}$ traces a two-dimensional path locus. Into this extension I shall not enter here.

The classification of plane collineations $(n=3)$ brings out little essentially new, but will be developed briefly to illustrate the method and to facilitate the discussion for $n=4$ which follows. It should, however, be pointed out that we are led to distinguish 9 classes of one-parameter groups in a plane, as against 7 previously noted. However, the two new classes (Nos. 5 and 8 ) are not real, even when the generating collineation $(m=1)$ is itself real.

$\S 11$. Collineations in a plane $(n=3)$.- - I shall classify a plane collineation $C$ simultaneously with the one-parameter group in which it is included. The equations of the former are obtained from those of the latter by putting $m=1$. The five types of each which are ordinarily distinguished are repeated below in normal form. By iteration further subdivision into classes results, which will be numbered consecutively and independently of the type number under which they are found. 
Type I. $x_{i}^{\prime}=\rho_{i}^{m} x_{i},(i=1,2,3), \rho_{i} \neq \rho_{j} . \quad$ Characterized by three invariant points and lines, which are taken to be the coördinate system. Assume $\left|\rho_{1}\right| \geqq\left|\rho_{2}\right| \geqq\left|\rho_{3}\right|$. Then on increasing and decreasing $m$ either through integral values or continuously we obtain a subdivision into the following 3 classes.

Class 1. $\left|\rho_{1}\right|>\left|\rho_{2}\right|>\left|\rho_{3}\right|$. - When $m$ starts from the value 0 and increases indefinitely, $P^{\prime}=\left(x_{1}^{\prime}, x_{2}^{\prime}, x_{3}^{\prime}\right)$ leaves an initial position $P=\left(x_{1}, x_{2}, x_{3}\right)$ and approaches as a limit the invariant point $I_{1}=(1,0,0)$, unless $P$ should lie upon the invariant line $x_{1}=0$ when the limit is the second invariant point $I_{2}=(0,1,0)$. For indefinite decrease of $m$ the limit of $P^{\prime}$ is the third invariant point $I_{3}=(0,0,1)$. When the $\rho_{i}$ are all real and of like sign, $C$ is generated by a real infinitesimal transformation and is said to be of hyperbolic type. The form of the path curves is well known.* A consideration of the ratios of $x_{1}, x_{1}, x_{3}$ for $m=+\infty$ or $-\infty$, will show that at $I_{1}$ and $I_{2}$ they are tangent to $x_{3}=0$ and $x_{1}=0$ respectively. When the $\rho_{i}$ are not all of the same sign, $C$ contains as a constituent a projective reflection, but the limiting effect of the iteration is not affected thereby.

Class 2. $\left|\rho_{1}\right|>\left|\rho_{2}\right|=\left|\rho_{3}\right|$.-No separate discussion is required for the assumption $\left|\rho_{1}\right|=\left|\rho_{2}\right|>\left|\rho_{3}\right|$, since the characteristic roots for our inverse substitution $C^{-1}$ have the order of magnitude $\left|\rho_{3}^{-1}\right|=\left|\rho_{2}^{-1}\right|>\left|\rho_{1}^{-1}\right|$. For indefinite increase of $m$ the point $P^{\prime}$ has the limit $I_{1}$, unless the initial point $P$ lies upon $x_{1}=0$, while for indefinite decrease of $m$ there is no limiting position of $P^{\prime}$. Real groups of this class are obtained only when the initial transformation $C^{\prime}$ which has been transformed into $C$ is real and gives a pair of conjugate imaginary roots $\rho_{2}, \rho_{3}$ for its characteristic equation (2). Then the real equations of the group have the form VI of $\S 9$, where $\rho_{1}$ may be taken to be positive. The path curves take their simplest form when the line at infinity is chosen for the axis $x_{1}=0$, and when the two invariant points upon it are taken to be the circular points. Then $y=0, z=0$ are at right angles, and the path curves form a system of logarithmic spirals winding around the origin.

Class 3. $\left|\rho_{1}\right|=\left|\rho_{2}\right|=\left|\rho_{3}\right|$. -The indefinite increase or decrease of $m$ gives no limiting position for $P^{\prime}$. When $C^{\prime}$ and its group are real, the latter has the real form VII given in $\S 9$. The logarithmic spirals of class 2 have now degenerated into concentric circles around the origin $y=0, z=0$.

Type II.

$$
\begin{aligned}
& x_{1}^{\prime}=\rho_{1}^{m} x_{1}, \\
& x_{2}^{\prime}=m \rho_{1}^{m-1} x_{1}+\rho_{1}^{m} x_{2}, \\
& x_{3}=\rho_{3}^{m} x_{3}
\end{aligned}
$$

Two invariant points, $I_{2}=(0,1,0), I_{3}=(0,0,1)$.

* For drawings and further information concerning the plane $W$-curves, see Scherfers, Encyclopaedie der math. Wissenschaften, III D4, \$\& 13-19. 
Class 4. $\left|\rho_{1}\right|>\left|\rho_{3}\right|$.- The indefinite increase and decrease of $m$ give $I_{2}$ and $I_{3}$ respectively as the limits of $P^{\prime}$. If $\rho_{1}, \rho_{3}$ are real and of same sign, the group is real. The system of path curves will be found in the second figure of SCHEFfers, our equations showing that they are tangent to $x_{3}=0$ and $x_{1}=0$ at $I_{2}$ and $I_{3}$ respectively.

Class 5. $\left|\rho_{1}\right|=\left|\rho_{3}\right|$. In this case $x_{1}^{\prime}, x_{3}^{\prime}$ tend with increasing $|m|$ to become infinitesimal in comparison with $x_{2}^{\prime}$. Hence whether $m$ increases or decreases, $P^{\prime}$ has a unique limiting position $I_{2}$. An exception occurs if the initial point $P$ lies upon the invariant line $x_{1}=0$, when there will be no limiting position for $P^{\prime}$. There is no real collineation group of this class, since $\rho_{1}, \rho_{3}$ are of opposite sign when real. For $m=1, \rho_{1}=-\rho_{3}$, we have as the initial collineation $C$ one which differs from the initial collineation of class 9 merely in the inclusion of a projective reflection. This prevents its generation by a real infinitesimal collineation.

Type III and class $6 . \quad x_{1}^{\prime}=\rho^{m} x_{1}$,

$$
\begin{aligned}
& x_{2}^{\prime}=m \rho^{m-1} x_{1}+\rho^{m} x_{2}, \\
& x_{3}^{\prime}=\frac{m(m-1)}{2 !} \rho^{m-2} x_{1}+m \rho^{m-1} x_{2}+\rho^{m} x_{3} .
\end{aligned}
$$

By cancellation of the common factor $\rho^{m}$ in the three right-hand members, it will be seen that the path curves are rational, the form of the equations showing that they are conics which touch the only invariant line $x_{1}=0$ at the only invariant point $(0,0,1)$. They have, moreover, quadruple contact with one another in this point.

Types IV and V are degenerate cases of I and II in which the path curves are straight lines.

Type IV. Same as I but with $\rho_{2}=\rho_{3}$.

IV, Class 7. $\left|\rho_{1}\right|>\left|\rho_{2}\right|$. - When $m$ indefinitely increases or decreases, $P^{\prime}$ moves on a line $x_{2}=c x_{3}$, and its two limiting positions are $(1,0,0)$ and the invariant intersection of this line with $x_{1}=0$.

Class 8. $\left|\rho_{1}\right|=\left|\rho_{2}\right|$. - There is no limiting position for $P^{\prime}$ on its path line. The group can not be real. $C$ is a projective reflection when $\rho_{1}=-\rho_{2}$.

Type $V$ and class 9 . Same as II but with $\rho_{1}=\rho_{3}$. The limit of $P^{\prime}$ on the invariant line $x_{1}=c x_{3}$ is the point $(0,1,0)$ irrespective of whether $m$ increases or decreases.

In conclusion, it may be pointed out that the collineations of classes 7 and 8 , namely

$$
x_{1}^{\prime}=\rho_{1} x_{1}, \quad x_{2}^{\prime}=\rho_{2} x_{2}, \quad x_{3}^{\prime}=\rho_{2} x_{3},
$$

can also be included in groups of class 2 or 3 respectively. By placing them, 
as has been done, in groups characterized by exactly the same invariant points rather than in groups with fewer such points, an exact parallelism between the classification of collineations and one-parameter collineation groups has been attained. When collineations of class 7 and 8 are real, they can, of course. be included in real groups of type VI and VII, $\$ 9$.

\section{$\S 12$. Collineations in three dimensions $(n=4)$.}

I shall, as before, classify simultaneously collineations and one-parameter collineation groups. The conditions characterizing types and classes will be given in their general form, but the discussion will be confined to the real representatives of the classes. Consequently throughout the discussion the $\rho_{i}$ are assumed to be positive or pairs of conjugate imaginaries. The principal aim in view is to find the real groups which give real $W^{\prime}$-curves. The possible types of real groups are denoted by $G_{i}(i=1,2, \cdots)$. By the iterative principle the 13 types of space collineations and one-parameter groups subdivide into 34 classes. In the last 16 of these classes the path curves lie in planes passing through a common axis, and in two others (11 and 17) the path curves are imaginary. I obtain therefore 16 real types of space $W$-curves.*

When invariant quadric surfaces can be formed under any class, this fact is noted.

Type I. $\quad x_{i}^{\prime}=\rho_{i}^{m} x_{i}, \quad \rho_{i} \neq \rho_{i} \quad(i=1,2,3,4)$.

It may be assumed that $\left|\rho_{1}\right| \geqq\left|\rho_{2}\right| \geqq\left|\rho_{3}\right| \geqq\left|\rho_{4}\right|$.

Class 1. $\left|\rho_{1}\right|>\left|\rho_{2}\right|>\left|\rho_{3}\right|>\left|\rho_{4}\right|$. - This case is parallel to case 1 of plane collineations. In each of the four invariant planes $x_{i}=0$ we obtain plane collineations. When $P^{\prime}=\left(x_{1}^{\prime}, \cdots, x_{4}^{\prime}\right)$ starts from any initial position $P=\left(x_{1}, \cdots, x_{4}\right)$ outside of these planes, it describes a true space curve terminating for $m=+\infty$ in the invariant point $I_{1}=(1,0,0,0)$ and for $m=-\infty$ in $I_{4}=(0,0,0,1)$. This particular class of $W$-curves is very well known and has the important property that their tangents cut the coördinate planes in four points whose anharmonic ratio is constant, not merely for a single path curve but for the entire system. $\dagger$ Consideration of ratios of the $x_{i}^{\prime}$ shows that the path curves at $I_{1}$ are tangent to the line $x_{3}=0$, $x_{4}=0$ and have $x_{4}=0$ for their osculating plane. A similar result holds at $I_{4}$.

* Scheffers (III D.IV, p. 214) seems to say that the number of varieties is 13 . Although this is the number of types of continuous one-parameter collineation groups when no account is taken of reality, several varieties or classes of $W$-curves are to be found under some of these types.

† Cf. KLein and Lie, Comptes Rendus, loc. cit. Other interesting properties are given. Their attention is confined for the most part to the curves belonging to class 1 . $C f$. also Scheffers, loc. cit., $\$ 20$. 
When $\rho_{1} \rho_{4}=\rho_{2} \rho_{3}$, the path curves lie upon the invariant hyperboloids $x_{1} x_{4}=$ cst. $x_{2} x_{3}$. Also if either $\rho_{2}^{2}$ or $\rho_{3}^{2}$ is equal to the product of two of the other three $\rho_{i}$, the curves will lie upon a surface of second order. If both $\rho_{1} \rho_{4}=\rho_{2} \rho_{3}$ and $\rho_{2}^{2}=\rho_{1} \rho_{3}$, then $\rho_{3}^{m} / \rho_{4}^{m}$ may be taken as a parameter $\tau$, in terms of which the equations of the path curves are

where

$$
x^{\prime}=\tau^{3} x, \quad y^{\prime}=\tau^{2} y, \quad z^{\prime}=\tau z,
$$

$$
x=x_{1} / x_{4}, \quad y=x_{2} / x_{4}, \quad z=x_{3} / x_{4} .
$$

Hence for this special case we have a system of twisted cubics, having contact in two of the invariant points.

Class 2. $\left|\rho_{1}\right|>\left|\rho_{2}\right|=\left|\rho_{3}\right|>\left|\rho_{4}\right|$.- To obtain real path curves we must suppose $\rho_{2}, \rho_{3}$ to be conjugate imaginaries and employ correspondingly two equations of form (14). We obtain thus a second type of real one-parameter group:

$$
\begin{aligned}
& x_{i}^{\prime}=\rho_{i}^{m} x_{i}, \\
& x_{j}^{\prime}=\rho_{j}^{m} x_{j} \\
& y_{1}^{\prime}=\sigma^{m}\left[y_{1} \cos m \vartheta-z_{1} \sin m \vartheta\right], \quad\left(\rho_{i} \neq \rho_{j}\right), \\
& z_{1}^{\prime}=\sigma^{m}\left[y_{1} \sin m \vartheta+z_{1} \cos m \vartheta\right],
\end{aligned}
$$

where in the particular case before us we have

$$
i=1, \quad j=4, \quad \rho_{i}=\rho_{1}, \quad \rho_{j}=\rho_{4}, \quad \sigma e^{i \vartheta}=\rho_{3}, \quad \sigma e^{-i \vartheta}=\rho_{2} .
$$

Here real coördinates $y_{1}, z_{1}$ are used in place of $x_{2}=y_{1}+i z_{1}, x_{3}=y_{1}-i z_{1}$. The form of the equations shows that as $m$ traverses its axis of reals positively or negatively, all path curves outside of the invariant planes tend toward the two real invariant points $I_{1}=(1,0,0,0)$ and $I_{4}=(0,0,0,1)$ respectively as poles, and wind an infinite number of times around the joining line.

To study the curves further take $x_{1}=0$ to be the plane at infinity and use ordinary orthogonal coördinates

$$
x=\frac{x_{j}}{x_{i}}, \quad y=\frac{y_{1}}{x_{i}}, \quad z=\frac{z_{1}}{x_{i}} .
$$

Then the path curves will lie upon the surfaces of revolution

$$
x^{2}=\operatorname{cst} .\left(y^{2}+z^{2}\right)^{k},
$$

where

$$
k=\frac{\log \rho_{j} / \rho_{i}}{\log \sigma / \rho_{i}} .
$$

This is generated by a plane $W$-curve $x=c r^{k / 2}$ of class 1 . Upon these surfaces of revolution the path curves wind around the $x$-axis in such a manner 
that their orthogonal projections upon $x=0$ are logarithmic spirals having the origin as pole.

A special case of interest is that in which $\sigma^{2}=\rho_{1} \rho_{4}$. Then the surfaces of revolution are paraboloids generated by the parabolas $x=c r^{2}$. We can collineate these into ellipsoids and hyperboloids having double contact with one another. If, in fact, we put (17) into the homogeneous form $x_{1} x_{4}=\operatorname{cst}$. [ $\left.y_{1}^{2}+z_{1}^{2}\right]$ and choose for $x_{1}=0$ and $x_{4}=0$ two parallel planes cut orthogonally by $y_{1}=0, z_{1}=0$ in the points $I_{4}$ and $I_{1}$ respectively, this will be accomplished. The ellipsoids of revolution lie between the parallel planes which they touch in $I_{4}$ and $I_{1}$, and among them is one which is a sphere. It can now be shown quickly that the $W$-curves upon the sphere are spherical loxodromes. To prove this, it suffices to show that seen from $I_{4}$ they are the stereographic projections upon the sphere of a set of logarithmic spirals which lie in the plane $x_{4}=0$ tangent to the sphere at the diametrically opposite point $I_{1}$ and have this point as their common pole. Now the equations of the cones which project the $W$-curves from $I_{4}$ as center are obtained from $G_{2}$ by omitting the equation $x_{4}^{\prime}=\rho_{4}^{m} x_{4}$. Consider the section of this cone with $x_{4}=0$. If the homogeneous coördinates receive the usual interpretation by which they are proportional to the distances of the point from the corresponding coördinate planes, $y_{1}^{\prime}$ and $z_{1}^{\prime}$ for any point of the section will be proportional to the distances of the point from the axis of $y_{1}$ and $z_{1}$ in this plane. The equations for $y_{1}^{\prime}, z_{1}^{\prime}$ show then that the section is a logarithmic spiral with the origin $I_{1}$ as pole. Hence the $W$-curves upon our family of ellipsoids and hyperboloids are their intersections with cones having these logarithmic spirals for their bases and $I_{4}$ for their common vertex. In particular, their intersections with the sphere are the desired loxodromes. This special case of a $W$-curve has been previously noted.*

Class 3.† $\left|\rho_{1}\right|>\left|\rho_{2}\right|>\left|\rho_{3}\right|=\left|\rho_{4}\right|$. - The real groups of this class have the above form $G_{2}$ but with the special values

$$
i=1, \quad j=2, \quad \rho_{3}=\sigma e^{i \vartheta}, \quad \rho_{4}=\sigma e^{-i \vartheta}
$$

The discussion is quite similar to that for class 2 . In the generating curve (17) we now have $k<1$, whereas in class 2 we have $k>1$. As before, the $W$-curves are spirals winding around the axes of $x$ and having $I_{1}$ as a pole, but the surfaces of revolution (17) upon which the spirals lie so spread away

* Cf. KLeIN and Lie, loc. cit., p. 1224.

$\dagger$ This case is identical with that for which $\left|\rho_{1}\right|=\left|\rho_{2}\right|>\left|\rho_{3}\right|>\left|\rho_{4}\right|$, since for the inverse substitution in class 3 the characteristic roots have the reverse order $\left|\rho_{4}^{-1}\right|=\left|\rho_{3}^{-1}\right|>\left|\rho_{2}^{-1}\right|>\left|\rho_{1}^{-1}\right|$. A corresponding remark applies to the other classes, and the reader will find that all possibilities have been duly cared for. 
from the $x$-axis as not to pass through $I_{4}$. This point is no longer a second asymptotic pole of the spirals.

Class 4. $\left|\rho_{1}\right|>\left|\rho_{2}\right|=\left|\rho_{3}\right|=\left|\rho_{4}\right|$.-We have the same equations as for class 3 but with $k=1$. Hence the surfaces (17) are cones of revolution. The $W$-spirals upon these cones are doubly loxodromic, cutting at one constant angle the pencil of planes through the axis of the cone and also at a constant angle a second pencil of planes parallel to $x=0 .^{*}$ The invariance of the two angles follows a consequence that the orthogonal differentials $d x^{\prime}$, $d r \equiv d \sqrt{\left(y^{\prime}\right)^{2}+\left(z^{\prime}\right)^{2}}$ and $\left(y^{\prime} d z^{\prime}-z^{\prime} d y^{\prime}\right) / \sqrt{\left(y^{\prime}\right)^{2}+\left(z^{\prime}\right)^{2}}$, due to any infinitesimal change in the parameter $m$, contain a common factor $\rho_{j}^{m} / \rho_{i}^{m}$, which is the only part dependent upon $m$.

Classes 5 and $6 . \quad\left|\rho_{1}\right|=\left|\rho_{2}\right|>\left|\rho_{3}\right|=\left|\rho_{4}\right|$. - To obtain a real group we must take $\rho_{1}, \rho_{2}$ and $\rho_{3}, \rho_{4}$ to be pairs of conjugate imaginaries. We introduce accordingly two sets of equations (14) and obtain the following type of real one-parameter group:

$$
\begin{array}{rlr}
y_{1}^{\prime} & =\sigma_{1}^{m}\left[y_{1} \cos m \vartheta_{1}-z_{1} \sin m \vartheta_{1}\right], & \\
z_{1}^{\prime}=\sigma_{1}^{m}\left[y_{1} \sin m \vartheta_{1}+z_{1} \cos m \vartheta_{1}\right] & \left(\rho_{1}=\sigma_{1} e^{i \vartheta_{1}}\right), \\
y_{2}^{\prime}=\sigma_{2}^{m}\left[y_{2} \cos m \vartheta_{2}-z_{2} \sin m \vartheta_{2}\right], & \\
z_{2}^{\prime}=\sigma_{2}^{m}\left[y_{2} \sin m \vartheta_{2}+z \cos m \vartheta_{2}\right] & \left(\rho_{2}=\sigma_{2} e^{i \vartheta_{2}}\right) .
\end{array}
$$

The four invariant points are now pairs of conjugate imaginaries which lie upon the two opposite invariant edges, $y_{1}=0, z_{1}=0$ and $y_{2}=0, z_{2}=0$, of our tetraedron of reference. Obviously $P^{\prime}$ lies in two planes revolving around these edges as axes. If, for convenience, these edges are taken perpendicular to each other and also the coördinate planes which meet in each, the two moving planes change uniformly with $m$, revolving at rates depending upon $\vartheta_{1}$ and $\vartheta_{2}$. At the same time the ratio of the distances $P^{\prime}$ from the two axes, $\sqrt{\left(y_{1}^{\prime}\right)^{2}+\left(z_{1}^{\prime}\right)^{2}} / \sqrt{\left(y_{2}^{\prime}\right)^{2}+\left(z_{2}^{\prime}\right)^{2}}$, increase in geometric ratio as $\sigma_{1}^{m} / \sigma_{2}^{m}$. While it may be said that in general the path curves wind simultaneously around the two edges, the statement may require proper modification because of the passage of $P^{\prime}$ to the plane at infinity during a revolution of either plane.

The characteristics of the path curves depend upon the relative commensurability or incommensurability of $\vartheta_{1}$ and $\vartheta_{2}$. We consequently distinguish the two following cases.

* Schefrers, loc. cit., p. 252. Cf. also Lie-SchefFers, Vorlesungen über Differentialgleichungen, p. 244. 
Class 5. $\quad \sigma_{1}>\sigma_{2} ; \vartheta_{2}$ and $\vartheta_{2}$ relatively commensurable.-Suppose $q \vartheta_{2}=p \vartheta_{1}$ where $p$ and $q$ are integers relatively prime. After a change of $q \pi / \vartheta_{1}$ in the value of $m$ the two revolving planes return to their initial positions, having performed $q$ and $p$ half revolutions respectively about their axes. Hence the path-spiral cuts an infinite number of times every intersection of the two revolving planes.

A special case of interest is that for which $\vartheta_{1}= \pm \vartheta_{2}$. Because the two planes revolve at the same rate, their intersections compose one set of generators of a ruled surface of second order. The axes of revolution belong to the other set and divide the surface into two regions. The generating point $P^{\prime}$ can not cross these axes since they are invariant lines for $G_{3}$ and must therefore remain continually in one of the two regions. This can only be if $P^{\prime}$ passes to infinity during each half-revolution of the two planes. The $W$-curve which it traces therefore traverses the region from infinity to infinity an infinite number of times. Furthermore, as $m$ indefinitely increases or decreases, $\sigma_{1}{ }^{m} / \sigma_{2}{ }^{m}$ tends toward $\infty$ or 0 , and $\overline{\left(y_{2}^{\prime}\right)^{2}+\left(z_{2}^{\prime}\right)^{2}}$ tends to become infinitesimal in comparison with $\sqrt{\left(y_{1}^{2}\right)+\left(z_{1}^{2}\right)}$ or vice versa. The branches of the $W$-curve tend to approximate in the one case to the axis $y_{2}=0, z_{2}=0$ and in the other case to $y_{1}=0, z_{1}=0$.

Class 6 . Same as class 5 but with $\vartheta_{1}, \vartheta_{2}$ relatively incommensurable.-The path curve meets each intersection of the two revolving planes but once.

Class 7. $\sigma_{1}=\sigma_{2} ; \vartheta_{1}, \vartheta_{2}$ unequal and relatively commensurable.-This case differs from case 5 , in that $P^{\prime}$ returns to its initial position after an increment of $\pm q \pi / \vartheta_{1}$ or $\pm 2 q \pi / \vartheta_{1}$ in $m$ according as $q-p$ is even or odd. Consequently the path curves now close. They may be regarded as degenerate cases of the unclosed spirals of class 5 , just as the circles of class 3 of plane collineations have been looked upon as degenerate cases of the logarithmic spirals of the preceding case.

Let $r$ denote the larger of the two numbers $|p|$ and $|q|$. Then if all the right hand members of $G_{3}$ be multiplied by $\sec ^{r} m \vartheta_{1} / q$, they can be expressed as polynomials in terms of $\tau=\tan m \vartheta_{1} / q$ or $\tau=\tan m \vartheta_{1} / 2 q$ according as $q-p$ is even or odd. It thus appears that the $W$-curves of this class are rational curves of degrees $r$ and $2 r$ respectively. Furthermore each curve lies upon a ruled invariant quadric

$$
\left(y_{1}^{\prime}\right)^{2}+\left(z_{1}^{\prime}\right)^{2}=k^{2}\left[\left(y_{2}^{\prime}\right)^{2}+\left(z_{2}^{\prime}\right)^{2}\right]
$$

where $k$ has the value $\sqrt{y_{1}^{2}+z_{1}^{2}} / v^{\prime} \overline{y_{2}^{2}+z_{2}^{2}}$ depending upon the initial point $P=\left(y_{1}, z_{1}, y_{2}, z_{2}\right)$. Two particular generators of this quadric belonging to opposite systems have the equations 
and

$$
\frac{y_{1}^{\prime}}{\sqrt{y_{1}^{2}+z_{1}^{2}}}-\frac{y_{2}^{\prime}}{\sqrt{y_{2}^{2}+z_{2}^{2}}}=0, \quad \frac{z_{1}^{\prime} 1}{\sqrt{y_{1}^{2}+z_{1}^{2}}}-\frac{z_{2}^{\prime}}{\sqrt{y_{2}^{2}+z_{2}^{2}}}=0
$$

$$
\frac{y_{1}^{\prime}}{v^{\prime} \frac{y_{2}^{\prime}}{y_{1}^{2}+z_{1}^{2}}}-\frac{z_{1}^{\prime}}{\sqrt{y_{2}^{2}+z_{2}^{2}}}=0, \quad \frac{z_{2}^{\prime}}{\sqrt{y_{1}^{2}+z_{1}^{2}}}+\frac{\overline{y_{2}^{2}+z_{2}^{2}}}{\sqrt{y^{2}}}=0 \text {. }
$$

Substituting here the values for $y_{1}^{\prime}, \cdots, z_{2}^{\prime}$ given in $G_{3}$ and putting

$$
\sin \alpha_{1}=\frac{y_{1}}{\sqrt{y_{1}^{2}+z_{1}^{2}}}, \quad \cos \alpha_{1}=\frac{z_{1}}{\sqrt{y_{1}^{2}+z_{1}^{2}}}, \quad \sin \alpha_{2}=\frac{y_{2}}{\sqrt{y_{2}^{2}+z_{2}^{2}}},
$$

we obtain the following pairs of equations for determining the intersections of the curve with the two generators:

$$
\sin \left(m \vartheta_{1}-\alpha_{1}\right)=\sin \left(m \vartheta_{2}-\alpha_{2}\right), \quad \cos \left(m \vartheta_{1}-\alpha_{1}\right)=\cos \left(m \vartheta_{2}-\alpha_{2}\right),
$$

and

$\sin m\left(\vartheta_{1}-\alpha_{1}\right)=\sin \left(m \vartheta_{2}-\alpha_{2}\right), \quad \cos \left(m \vartheta_{1}-\alpha_{1}\right)=-\cos \left(m \vartheta_{2}-\alpha_{2}\right)$.

These have respectively the solutions

and

$$
\frac{m \vartheta_{1}}{q}=\frac{\alpha_{1}-\alpha_{2}}{q-p}+\frac{2 n \pi}{q-p} \quad(n=0,1,2, \cdots)
$$

$$
\frac{m \vartheta_{1}}{q}=\frac{\pi+\alpha_{1}+\alpha_{2}}{q+p}+\frac{2 n \pi}{q+p}
$$

When $q-p$ is given, there are $|q-p| / 2$ of the former solutions and $|q+p| / 2$ of the latter which lie within an interval of $\pi$ for $m \vartheta_{1} / q$. Hence the curves then intersect one system of generators of the quadric in $|q-p| / 2$ points and the other in $|q+p| / 2$ points. When $q-p$ is odd, these numbers are to be doubled.

Class 8. $\sigma_{1}=\sigma_{2} ; \vartheta_{1}$ and $\vartheta_{2}$ unequal and relatively incommensurable.The curves lie upon the ruled quadrics of class 7 , but they are no longer rational and they cut each generator an infinite number of times.

Type $I I$.

$$
\begin{aligned}
x_{1}^{\prime} & =\rho_{1}^{m} x_{1}, \\
x_{2}^{\prime} & =m \rho_{1}^{m-1} x_{1}+\rho_{1}^{m} x_{2}, \\
x_{3}^{\prime} & =\rho_{3}^{m} x_{3}, \\
x_{4}^{\prime} & =\rho_{4}^{m} x_{4}
\end{aligned}
$$


There are three invariant points: $I_{1}=(0,1,0,0), I_{2}=(0,0,1,0)$, $I_{3}=(0,0,0,1)$. When $|m|$ increases indefinitely, $x_{1}$ becomes infinitesimal in comparison with $x_{2}^{\prime}$. The nature of the path curves can best be seen by using the rectangular coördinates $x=x_{2} / x_{1}, y=x_{3} / x_{1}, z=x_{4} / x_{1}$. The equations of the curves become

$$
x^{\prime}=\frac{m}{\rho_{1}}+x, \quad y_{1}^{\prime}=\left(\frac{\rho_{3}}{\rho_{1}}\right)^{m} y, \quad z^{\prime}=\left(\frac{\rho_{4}}{\rho_{1}}\right)^{m} z .
$$

The point $P^{\prime}$ traces a $W$-curve on a cylinder whose axis is parallel to the $x$-axis and whose intersection with the $(y, z)$-plane is a plane $W$-curve of class 1. The position of these $W$-curves relatively to the invariant points depends upon the inequalities subsisting between the moduli of the $\rho_{i}$.

Class 9. $\left|\rho_{1}\right|>\left|\rho_{3}\right|>\left|\rho_{4}\right|$. - The path curves run between $I_{1}$ and $I_{3}$, having at $I_{1}$ a common tangent line, $x_{3}=0, x_{4}=0$, and a common osculating plane $x_{4}=0$ while at $I_{3}$ they are tangent to the opposite edge of the coördinate tetraedron and have $x_{1}=0$ as their common osculating plane. When $\rho_{3}^{2}=\rho_{1} \rho_{4}$, the curves lie upon the invariant quadric surfaces $x_{3}^{2}=c x_{1} x_{4}$.

Class 10. $\left|\rho_{3}\right|>\left|\rho_{1}\right|>\left|\rho_{4}\right|$. The path curves run between $I_{2}$ and $I_{3}$, having at each point an invariant coördinate axis as a common tangent and an invariant coördinate plane for osculating plane. When $\rho_{1}^{2}=\rho_{3} \rho_{4}$, they lie upon the quadric $x_{1}^{2}=c x_{3} x_{4}$.

Class 11. $\left|\rho_{1}\right|=\left|\rho_{3}\right|>\left|\rho_{4}\right|$. The group and path curves are imaginary.

Class 12. $\left|\rho_{1}\right| \neq\left|\rho_{3}\right|=\left|\rho_{4}\right|$.-Taking $\rho_{3}, \rho_{4}$ to be conjugate imaginaries, we have the following fifth type of real one-parameter group:

$$
\begin{aligned}
& x_{1}^{\prime}=\rho_{1}^{m} x_{1}, \\
& x_{2}^{\prime}=m \rho_{1}^{m-1} x_{1}+\rho_{1}^{m} x_{2},
\end{aligned}
$$

$$
\begin{array}{ll}
y_{1}^{\prime} & =\sigma^{m}\left[y_{1} \cos m \vartheta-z_{1} \sin m \vartheta\right], \\
z_{1}^{\prime} & =\sigma^{m}\left[y \sin m \vartheta+z_{1} \cos m \vartheta\right] \quad\left(\sigma e^{i \vartheta}=\rho_{8}\right)
\end{array}
$$

If we use the rectangular coördinates $x=x_{2} / x_{1}, y=y_{2} / x_{1}, z=z_{2} / x_{1}$, then in place of the last two equations of (18) we have two equations similar to the last two of $G_{5}$. Hence the path curves now lie upon cylinders whose cross sections are logarithmic spirals (i. e., plane $W$-curves of class 2 ).

Class 13. $\left|\rho_{1}\right|=\left|\rho_{3}\right|=\left|\rho_{4}\right|$. - Since $\rho_{1}=\sigma$, a common factor $\sigma^{m}$ can be removed from all four equations of $G_{5}$. The logarithmic cross sections of 
the cylinders of the preceding case become concentric circles. All the $W$-curves are ordinary helices with a common axis.*

Type III.

$$
\begin{array}{ll}
x_{1}^{\prime}=\rho_{1}^{m} x_{1}, & x_{3}^{\prime}=\rho_{3}^{m} x_{3}, \\
x_{2}^{\prime}=m \rho_{1}^{m-1} x_{1}+\rho_{1}^{m} x_{2}, & x_{4}^{\prime}=m \rho_{3}^{m-1} x_{3}+\rho_{3}^{m} x_{4}
\end{array}
$$

Using the same rectangular coördinates as under type II, we have in place of the last two equations for $y^{\prime}, z^{\prime}$ in (18) two others which are similar to those just given for $x_{3}^{\prime}, x_{4}^{\prime}$. The cross section of our cylinder is therefore now a plane $W$-curve of class 4 .

Class 14. $\left|\rho_{1}\right| \neq\left|\rho_{3}\right|$. - The path curves run between the only two invariant points $(0,1,0,0)$ and $(0,0,0,1)$, where they touch two invariant lines; etc.

Class 15. $\left|\rho_{1}\right|=\left|\rho_{3}\right|$. -Taking $\rho_{1}, \rho_{3}$ to be conjugate imaginaries we get the following seventh type of real group:

$$
\begin{aligned}
& y_{1}^{\prime}=\sigma^{m}\left[y_{1} \cos m \vartheta-z_{1} \sin m \vartheta\right], \quad z_{1}^{\prime}=\sigma^{m}\left[y_{1} \sin m \vartheta+z_{1} \cos m \vartheta\right], \\
& y_{2}^{\prime}=m \sigma^{m-1}\left[y_{1} \cos (m-1) \vartheta-z_{1} \sin (m-1) \vartheta\right] \\
& +\sigma^{m}\left[y_{2} \cos m \vartheta-z_{2} \sin m \vartheta\right], \\
& z_{2}^{\prime}=m \sigma^{m-1}\left[y_{1} \sin (m-1) \vartheta+z_{1} \cos (m-1) \vartheta\right] \\
& +\sigma^{m}\left[y_{2} \sin m \vartheta+z_{2} \cos m \vartheta\right] .
\end{aligned}
$$

For convenience, take the non-intersecting axes $y_{1}=0, z_{1}=0$ and $y_{2}=0$, $z_{2}=0$ to be perpendicular. Then $P^{\prime}$ lies in two planes revolving about these axes. One of these planes turns uniformly about the former axis, for uniform change in $m$, but the motion of the other plane depends on two groups of terms of rotary character in $y_{2}^{\prime}, z_{2}^{\prime}$. One of these groups is affected with a multiplier $m$, and hence as $|m|$ increases indefinitely, the other group becomes relatively infinitesimal. In consequence, the motion of the second plane about its axis tends to become uniform. Now an increment of $\pi / \vartheta$ in $m$ will change simultaneously the signs of all four coördinates of $P^{\prime}$ and for excessively great values of $|m|$ alters the position of $P^{\prime}$ only infinitesimally. But it also turns the first plane through a half revolution about its axis $y_{1}=0$, $z=0$. As $P^{\prime}$ can not cross this invariant axis and returns very nearly to its initial position after the half rotation, it must have passed out to infinity in the

* Cf. Schermers, loc. cit., p. 215. 
course of the half rotation. Furthermore, as $|m|$ increases indefinitely, $y_{1}^{\prime}, z^{\prime}$ tend to become infinitesimal in comparison with $\sqrt{\left(y_{2}^{\prime}\right)^{2}+\left(z_{2}^{\prime}\right)^{2}}$. We see therefore that our path curve consists of an infinity of pieces which with indefinitely increasing or decreasing $m$ tend to approximate to the only invariant real line of the collineation, $y_{1}=0, z_{1}=0$.

If $\rho_{1}=-\rho_{3}$ our path curves are imaginary but lie upon invariant quadrics, $x_{2} x_{3}+x_{1} x_{4}+c x_{1} x_{3}=0$.

Type IV. $\quad x_{1}^{\prime}=\rho_{1}^{m} x_{1}$,

$$
\begin{aligned}
& x_{2}^{\prime}=m \rho_{1}^{m-1} x_{1}+\rho_{1}^{m} x_{2}, \\
& x_{3}^{\prime}=\frac{m(m-1)}{2} \rho_{1}^{m-2} x_{1}+m \rho_{1}^{m-1} x_{2}+\rho_{1}^{m} x_{3}, \\
& x_{4}^{\prime}=\rho_{4}^{m} x_{4}
\end{aligned}
$$$$
\left(\rho_{4} \neq \rho_{1}\right) .
$$

Class 16. $\left|\rho_{1}\right| \neq\left|\rho_{4}\right|$. - The path curves connect the only two invariant points $(0,0,1,0)$ and $(0,0,0,1)$, at each of which they touch one of the two invariant lines and osculate one of the two invariant planes. If rectangular coördinates are introduced as under type II, they will lie upon cylinders whose cross sections are conics (cf. class 6 of plane collineations).

Class 17. $\left|\rho_{1}\right|=\left|\rho_{4}\right|$. - The path curves are imaginary, and have the point $(0,0,1,0)$ as terminus or limiting point, irrespective of whether $m$ increases or decreases indefinitely.

Type $V$ and class 18.

$$
\begin{aligned}
x_{1}^{\prime} & =\rho^{m} x_{1}, \\
x_{2}^{\prime} & =m \rho^{m-1} x_{1}+\rho^{m} x_{2}, \\
\left(G_{9}\right) \quad x_{3}^{\prime} & =\frac{m(m-1)}{2 !} \rho^{m-2} x_{1}+m \rho^{m-1} x_{2}+\rho^{m} x_{3}, \\
x_{4}^{\prime} & =\frac{m(m-1)(m-2)}{3 !} \rho^{m-3} x_{1}+\frac{m(m-1)}{2 !} \rho^{m-2} x_{2}+m \rho^{m-1} r_{3}+\rho^{m} x_{4} .
\end{aligned}
$$

After cancellation of the common factor $\rho^{m}$ we have polynomials in $m$ of degree $\leqq 3$, which shows that the $W$-curves are twisted cubics. They pass through the only invariant point where they touch the only invariant straight line and osculate the only invariant plane. As in type IV, the curves lie upon invariant cones defined by the first three equations. 
This completes the enumeration of the cases in which the $W$-curves are true space curves. In the remaining types of collineations two or more of the $\rho_{i}$ of the component collineations are equal. If $\rho_{i}=\rho_{j}$, we have a pair of equations of form $x_{i}=\rho_{i}^{m} x_{i}, x_{j}^{\prime}=\rho_{j}^{m} x_{j}$, so that the path curves must lie in the invariant planes of the pencil $x_{i}=c x_{j}$. In the remaining classes the systems of path curves differ from one another in respect to the disposition and types of the plane $W$-curves involved. The classes will be enumerated without further comment.

Type VI. Same as I with $a_{\rho_{i}}$ and $\rho_{j}$ equal.

Class 19. $\quad \rho_{1}=\rho_{2} ; \quad\left|\rho_{1}\right|>\left|\rho_{3}\right|>\left|\rho_{4}\right|$.

Class 20. $\rho_{2}=\rho_{3} ; \quad\left|\rho_{1}\right|>\left|\rho_{2}\right|>\left|\rho_{3}\right|$.

Class 21. $\quad \rho_{1}=\rho_{2} ; \quad\left|\rho_{1}\right|=\left|\rho_{3}\right|>\left|\rho_{4}\right|$.

Class 22. $\rho_{1}=\rho_{2} ; \quad\left|\rho_{1}\right| \neq\left|\rho_{3}\right|=\left|\rho_{4}\right|$. -This gives an additional type of real group, the same as $G_{2}$ but with $\rho_{i}=\rho_{j}$.

Class 23. $\rho_{1}=\rho_{2} ; \quad\left|\rho_{1}\right|=\left|\rho_{3}\right|=\left|\rho_{4}\right|$.

Type VII. Same as I but with $\rho_{1}=\rho_{2}=\rho_{3}$.

Class 24. $\left|\rho_{1}\right| \neq\left|\rho_{4}\right|$.

Class 25. $\left|\rho_{1}\right|=\left|\rho_{4}\right|$.

Type VIII. Same as I but with $\rho_{1}=\rho_{2} \neq \rho_{3}=\rho_{4}$.

Class 26. $\left|\rho_{1}\right| \neq\left|\rho_{3}\right|$.

Class 27. $\left|\rho_{1}\right|=\left|\rho_{3}\right|$. - This gives another type of real group, the same as $G_{3}$ but with $\sigma_{1} e^{s_{1}}=\sigma_{2} e^{s_{3}}$.

Type IX. Same as II but with $\rho_{1}=\rho_{3}$.

Class 28. $\left|\rho_{1}\right| \neq\left|\rho_{4}\right|$.

Class 29. $\left|\rho_{1}\right|=\left|\rho_{4}\right|$.

Type $X$. Same as II but with $\rho_{3}=\rho_{4}$.

Class 30. $\left|\rho_{1}\right| \neq\left|\rho_{3}\right|$.

Class 31. $\left|\rho_{1}\right|=\left|\rho_{3}\right|$.

Type $X I$ and class 32. Same as II but with $\rho_{1}=\rho_{3}=\rho_{4}$.

Type XII and class 33. Same as III but with $\rho_{1}=\rho_{3}$.

Type XIII and class 34. Same as IV but with $\rho_{1}=\rho_{4}$.

These 8 types furnish 10 types of real groups, making 19 in all.

\$13. The associated quadratic complexes.-It is well known that in the case of the one-parameter group of type $I, \S 11$, the tangents to its path curves form a quadratic complex, commonly called the tetraedral complex. The six line coördinates of the tangent to the path curve through $\left(x_{1}^{\prime}, \cdots, x_{4}^{\prime}\right)$ are evidently given by expressions of the form 
$p_{i j} \equiv\left|\begin{array}{cc}x_{i}^{\prime} & x_{j}^{\prime} \\ x_{i}^{\prime}+d x_{i}^{\prime} & x_{j}^{\prime}+d x_{j}\end{array}\right|=\left|\begin{array}{cc}x_{i}^{\prime} & x_{j}^{\prime} \\ d x_{i}^{\prime} & d x_{j}^{\prime}\end{array}\right|=\left|\begin{array}{cc}\rho_{i}^{m} x_{i} & \rho_{j}^{m} x_{j} \\ \rho_{i}^{m} x_{i} \log \rho_{i} & \rho_{j}^{m} x_{j} \log \rho_{j}\end{array}\right|$

$$
=x_{i} x_{j} \rho_{i}^{m} \rho_{j}^{m} \log \frac{\rho_{j}}{\rho_{i}} .
$$

They accordingly satisfy the quadratic equation

$$
A p_{12} p_{34}+B p_{13} p_{24}+C p_{14} p_{23}=0 \text {, }
$$

when the coefficients are connected by the relation

$$
A \log \frac{\rho_{2}}{\rho_{1}} \log \frac{\rho_{4}}{\rho_{3}}+B \log \frac{\rho_{3}}{\rho_{1}} \log \frac{\rho_{4}}{\rho_{2}}+C \log \frac{\rho_{4}}{\rho_{1}} \log \frac{\rho_{3}}{\rho_{2}}=0
$$

The coördinates of the tangent lines of the path curves for types II-V can be found in similar manner. They furnish quadratic complexes of the following types:

II.

where

$$
A p_{13} p_{14}+B p_{12} p_{34}=0
$$

$$
A \log \frac{\rho_{3}}{\rho_{1}} \log \frac{\rho_{4}}{\rho_{1}}+\frac{B}{\rho} \log \frac{\rho_{4}}{\rho_{3}}=0 .
$$

III.

where

$$
A p_{13}^{2}+B p_{12} p_{34}=0 \text {, }
$$

$$
A\left[\log \frac{\rho_{3}}{\rho_{1}}\right]^{2}+\frac{B}{\rho_{1} \rho_{3}}=0 .
$$

IV.

where

$$
A\left(p_{12} p_{24}-p_{13} p_{14}\right)+B p_{12} p_{14}=0 \text {, }
$$

$$
A\left(-\frac{1}{\rho_{1}}+\frac{1}{2 \rho_{1}} \log \frac{\rho_{4}}{\rho_{1}}\right)+B \log \frac{\rho_{4}}{\rho_{1}}=0 .
$$

V.

$$
p_{12}\left(p_{23}+p_{14}\right)-p_{13}^{2}-\frac{1}{12 \rho^{2}} p_{12}^{2}=0
$$

The apparent form of these equations can be modified by means of the identity $p_{12} p_{34}+p_{13} p_{42}+p_{14} p_{23}=0$. Each equation and the identity together constitute a pair of quadratic forms whose elementary divisors serve to classify the complex. The characteristics of the five types will be seen to be respectively [(11) (11) (11)], [ (22) (11)], [ (211) (11) ], [(33)], [ (321) ]. The " singular surface" of the complex for Type I consists of the four invariant coördinate planes. This invariant tetraedron degenerates in the four succeeding types, but the singular surface comprises those of the coördinate planes which are invariant. 
For types VI-XIII the tangents to the path curves form a special linear complex, inasmuch as the path curves lie in a pencil of planes and accordingly their tangents meet the axis of the pencil. We conclude therefore that there are just 5 types of quadratic complexes which consist of the tangents to the path curves of a continuous one-parameter collineation group. As under our first 5 types of one parameter groups we distinguished various classes of real groups, so under our 5 types of quadratic complexes we may distinguish corresponding classes of real complexes, whose real equations are easily found.

Madison, Wisconsin. 